\title{
Clinical Characteristics and Factors Associated with Heart Failure Readmission at a Tertiary Hospital in North-Eastern Tanzania
}

\author{
Abid M. Sadiq $\mathbb{D}^{1},{ }^{1}$ Nyasatu G. Chamba $\mathbb{D}^{1,2}$ Adnan M. Sadiq, ${ }^{3}$ Elichilia R. Shao $\mathbb{D}^{1,2}$ \\ and Gloria A. Temu ${ }^{1,2}$ \\ ${ }^{1}$ Department of Internal Medicine, Kilimanjaro Christian Medical University College, Moshi, Tanzania \\ ${ }^{2}$ Department of Internal Medicine, Kilimanjaro Christian Medical Centre, Moshi, Tanzania \\ ${ }^{3}$ Department of Radiology, Kilimanjaro Christian Medical Centre, Moshi, Tanzania
}

Correspondence should be addressed to Abid M. Sadiq; abid.physician@gmail.com

Received 6 January 2020; Revised 20 February 2020; Accepted 18 April 2020; Published 30 April 2020

Academic Editor: Anne Knowlton

Copyright ( 92020 Abid M. Sadiq et al. This is an open access article distributed under the Creative Commons Attribution License, which permits unrestricted use, distribution, and reproduction in any medium, provided the original work is properly cited.

Introduction. Heart failure (HF) is characterized by frequent episodes of decompensation, leading to a high hospitalization burden. More than $50 \%$ of index hospitalizations for HF patients return within 6 months of discharge. Once the patient is readmitted, the risk of further disease progression and the mortality rate are increased. A lot of patients are readmitted due to factors such as poor medication adherence, infections, or worsening comorbidities. The aim of our study was to identify the inpatient burden of HF readmission and to identify the factors associated with early readmission. Methods. A hospital-based cross-sectional analytical study was conducted from November 2018 to April 2019 within the medical wards at Kilimanjaro Christian Medical Centre (KCMC), which is a teaching and referral hospital in north-eastern Tanzania. The study population included all patients with HF admitted within the medical ward. Data were collected using questionnaires and blood and radiological investigations, and analysis was done using Statistical Package for Social Science (SPSS) version 25. Chi-square test was used to compare proportions of categorical variables. Logistic regression was used to determine the likelihood for readmission, and $p$-value of $<0.05$ was considered to be statistically significant. Results. A total of 353 patients were identified with HF, of whom $136(38.5 \%)$ had a previous admission. Of the 136 patients analysed, the mean age was 62.8 years (SD 17.1), and $86(63.2 \%)$ were females. Within 30 days after discharge, $34(25.0 \%)$ of the patients were readmissions. Factors for early readmission were unemployment $(\mathrm{OR}=2.38,95 \% \mathrm{CI}=1.02-5.54, p=0.043)$, poor medication adherence $(\mathrm{OR}=3.87,95 \% \mathrm{CI}=1.67-8.97$, $p=0.002)$, absence of angiotensin-converting enzyme inhibitor (ACEI) or angiotensin receptor blocker $(\mathrm{ARB})(\mathrm{OR}=2.40,95 \%$ $\mathrm{CI}=1.09-5.31, p=0.030$ ), and pleural effusion (OR 3.25, 95\% CI $=1.44-7.32, p=0.004)$. Conclusion. Heart failure is a burden due to a large number of admissions and readmissions. Factors such as poor medication adherence and absence of adequate HF therapy, especially the absence of regimes containing ACEI or ARB, need to be targeted to reduce the number of readmissions. This will help reduce the risk of further decompensations, disease progression, and mortality rate.

\section{Introduction}

Heart failure (HF) is a major health problem worldwide and emerging in sub-Saharan Africa (SSA). Although there is lacking evidence on prevalence and incidence in the region, the identified causes include hypertension, cardiomyopathies, and rheumatic heart disease, but rarely ischemic heart disease $[1-3]$.

The identified causes inflict cardiac injury resulting in loss of myocyte function or impaired myocardial pumping force.
This leads to reduced cardiac output, and compensatory mechanisms are activated to improve function. However, the sustained activation of these systems can lead to secondary damage causing worsening left ventricular remodelling and cardiac decompensation thus resulting in HF [4]. This decompensation can lead to hospitalization, readmission, or death. Heart failure readmission is defined as an acute and unexpected admission to the same health centre after hospitalization for HF [5]. Early readmission is returning to the hospital within 30 days from the previous hospitalization [6]. 
Hospital readmissions and mortality rates can be used as an indicator of hospital quality since possibilities of prevention and control exist. Patients discharged from the hospital and readmitted within a short period of time are a cause of concern for the hospital [7]. Apart from hospital quality, socioeconomic factors such as poverty, residing in low-income neighbourhoods, or lack of social support have been observed as factors associated with readmission $[8,9]$.

The high rate of readmission and high treatment costs constitute an economic burden to patients and to health systems [2]. In 2014, it was estimated over $\$ 100$ billion was spent for HF globally, and Tanzania's estimated cost was \$19 million [10]. In Nigeria, the cost per patient per year was calculated to be $\$ 2,128$ [11].

Heart failure is difficult to treat and increasingly common with many patients being frequently admitted [12]. The reason patients get readmitted is due to various factors such as poor medication adherence, worsening comorbidities, inappropriate diet control, and infections [13]. Evidencebased therapies have been shown to reduce readmission and mortality in patients with impaired left ventricular ejection fraction, while to date no effective therapies are available to improve the survival of heart failure with preserved ejection fraction (HFpEF) [14].

The purpose of this study was to identify the inpatient burden of HF readmission as well as to identify the factors for early readmission. The clinical characteristics associated with heart failure readmission were also identified.

\section{Materials and Methods}

A hospital-based cross-sectional analytical study was carried out from November 2018 to April 2019 in the medical wards at Kilimanjaro Christian Medical Centre (KCMC), which is a consultant referral hospital in north-eastern Tanzania. The medical ward at KCMC admits patients from the age of 14 and above.

Permission to conduct the study was obtained from Kilimanjaro Christian Medical University College Research and Ethics Committee (no. 2315). Written consent (signature or fingerprint thumb) was obtained from the patients after being informed about the purpose and benefits of the study. Patients below the age of 18 years were informed and consent was obtained from their parents or guardians. For patients who were unable to provide consent, a relative provided consent. Confidentiality was observed and all data were stored unlinked to patient identifiers.

Patients were identified and detailed information was collected consecutively. A face to face interview was conducted, and data were entered into the questionnaire. Questionnaires had an identification (ID) number and not the name of the patient to abide by confidentiality. Data were collected on sociodemographic characteristics, disease associated information, and clinical characteristics. Clinical examination was performed to assess for the blood pressure (BP), pulse rate, respiratory rate, and temperature. Cardiovascular examination was performed to assess for jugular venous pressure, precordial hyperactivity, cardiac apex, and heart sounds. Medication adherence was assessed using the
Morisky Green Levine scale which asks 4 questions regarding the current medication use. Scoring 0 shows high adherence, while scoring 1-2 shows moderate adherence and 3-4 shows low adherence [15]. Physical activity was assessed by using the International Physical Activity Questionnaire, which assesses the metabolic equivalent task minutes (METminutes) per week. Scoring <600 MET-minutes shows low activity, while scoring $\geq 600$ to $<3,000$ MET-minutes shows moderate activity and $\geq 3,000$ MET-minutes shows high activity [16].

A standard procedure was used to measure the BP using a manual sphygmomanometer and temperature using a digital thermometer. Blood sample was collected with a $5 \mathrm{cc}$ syringe. From the syringe, $2 \mathrm{cc}$ of blood was put into a purple top EDTA vacutainer for full blood picture, and $3 \mathrm{cc}$ was put into a red top vacutainer for serum sodium, serum potassium, serum urea, serum creatinine, and serum cholesterol. The full blood picture sample was run through the Mindray BC 3200 (Gtech Medical, Brisbane, QLD, Australia). The biochemistry samples went through the Cobas Integra 400 Plus (Roche Diagnostics Ltd, CH-6343 Rotkreuz, Switzerland). Glucoplus (Glucoplus Inc, Saint Laurent, QC H4S 1S3 Canada) glucometer was used for assessment of patient's random blood glucose. Anaemia was identified by a reduction in haemoglobin less than $10.0 \mathrm{~g} / \mathrm{dL}$. Leucocytosis was identified by an elevated white blood cell count greater than $11.0 \times 10^{9}$ per L [17].

The radiological investigations for chest X-ray, to look for features of $\mathrm{HF}$, and echocardiography, for ejection fraction were done. The results were obtained from the Philips HD6 (Philips Electronics Nederland B.V., Boschdijk 525, 5621JG, Eindhoven, the Netherlands) machine, and the results were interpreted by a qualified radiologist. Electrocardiogram (ECG) was performed by a qualified nurse and interpreted by the principal investigator (PI).

Data were coded and entered into the Statistical Package for Social Science version 25 (SPSS V25). Data were examined for distribution and outliers. Descriptive analysis was used generating means and standard deviations for quantitative data and frequency distributions for categorical data. Chi-Square test was used to compare proportions of categorical variables. The crude odds ratio with its confidence interval of $95 \%$ was used to determine factors associated with increased likelihood of readmission. Achieving statistical significance meant that the probability $(p)$ value appreciated is $<0.05$. Potential confounders were controlled for by performing multivariate logistic regression analysis.

\section{Results}

A total of 353 patients were identified with $\mathrm{HF}$ and 136 (38.5\%) were identified as readmissions. The results displayed in Table 1 show the majority of the patients were females $(63.2 \%)$. The mean age was 62.8 years (SD 17.1), with $36(26.5 \%)$ being above the age of 75 years. Those unemployed were $102(75.0 \%)$ and without health insurance were $71(52.2 \%)$.

Of the 136 patients with readmission for $\mathrm{HF}, 34$ (25.0\%) readmitted within 30 days and 33 (24.3\%) were readmitted 
TABLE 1: Sociodemographic characteristics of patients readmitted for heart failure $(N=136)$.

\begin{tabular}{|c|c|c|c|c|c|}
\hline \multicolumn{6}{|c|}{ Readmission } \\
\hline Characteristics & $\begin{array}{c}\text { Total } \\
n(\%)\end{array}$ & $\begin{array}{c}\leq 30 \text { days } \\
n(\%)\end{array}$ & $\begin{array}{c}>30 \text { days } \\
n(\%)\end{array}$ & $\chi^{2}$ & $p$ value \\
\hline Mean age (SD) & $62.8(17.1)$ & $59.9(17.9)$ & $63.8(16.9)$ & & \\
\hline Age $>75$ years & $36(26.5)$ & $8(23.5)$ & $28(27.5)$ & 1.87 & 0.521 \\
\hline Female & $86(63.2)$ & $19(55.9)$ & $67(65.7)$ & 1.05 & 0.306 \\
\hline Rural setting & $114(83.8)$ & $29(85.3)$ & $85(83.3)$ & 0.07 & 0.788 \\
\hline Unemployed & $102(75.0)$ & $21(61.8)$ & $81(79.4)$ & 4.23 & 0.043 \\
\hline Not insured & $71(52.2)$ & $17(50.0)$ & $54(52.9)$ & 0.08 & 0.766 \\
\hline Hypertension & $87(64.0)$ & $18(52.9)$ & $69(67.6)$ & 2.39 & 0.122 \\
\hline Diabetes & $38(27.9)$ & $8(23.5)$ & $30(29.4)$ & 0.43 & 0.508 \\
\hline Absence of ACEI or ARB & $58(42.6)$ & $20(58.8)$ & $38(37.3)$ & 4.85 & 0.030 \\
\hline Absence of loop diuretic & $23(16.9)$ & $9(26.5)$ & $14(13.7)$ & 2.94 & 0.086 \\
\hline Poor medication adherence & $73(53.7)$ & $10(29.4)$ & $63(61.8)$ & 10.73 & 0.002 \\
\hline Poor physical activity & $103(75.7)$ & $27(79.4)$ & $76(74.5)$ & 0.33 & 0.794 \\
\hline Poor clinic visit & $75(55.1)$ & $23(67.6)$ & $52(51.0)$ & 2.86 & 0.094 \\
\hline
\end{tabular}

after $>1$ ear. Among the 136 patients, 73 (53.7\%) patients had poor medication adherence. The majority $(75.7 \%)$ did not have any sort of physical activity. Seventy-five (55.1\%) of the 136 patients did not attend regular clinic at KCMC.

Looking at their clinical histories, hypertension was seen in $87(64.0 \%)$ patients, and diabetes in 38 (27.9\%) patients. Twenty-three (16.9\%) patients were not using loop diuretics, and 58 (42.6\%) were not using angiotensin-converting enzyme inhibitor (ACEI) or angiotensin receptor blocker (ARB).

Seventy-two (52.9\%) patients readmitted were classified as New York Heart Association (NYHA) Class III. Elevated systolic blood pressure (SBP) was observed in 50 (36.8\%) patients and elevated diastolic blood pressure (DBP) was observed in 42 (30.9\%) patients. On cardiovascular examination, poor capillary refill was seen among 13 (9.6\%) patients and cardiac murmur was detected by auscultation in $47(34.6 \%)$ patients, as seen in Table 2.

Laboratory findings show that $23(16.9 \%)$ patients had leucocytosis, 26 (19.1\%) had anaemia and 78 (57.4\%) had an estimated glomerular filtration rate $(\mathrm{eGFR})<60$. On chest radiography, pleural effusion was seen among 41 (30.1\%) patients and $63(46.3 \%)$ had opacities consistent with pneumonia. Atrial fibrillation (AF) was seen in $42(23.5 \%)$ patients. The left ventricle findings showed HFpEF in 60 (44.1\%) patients, and heart failure with reduced ejection fraction (HFrEF) in 52 (38.2\%) patients. The left ventricle wall was dilated in 38 (27.9\%) patients. Mitral regurgitation (MR) was seen in $70(51.5 \%)$ patients.

Of the sociodemographic data, patients who were unemployed had an odds ratio (OR) of $2.38 \quad(95 \%$ $\mathrm{CI}=1.02-5.54)$ for being readmitted within 30 days $(p=0.043)$. Patients who had poor adherence to medication therapy had an OR of 3.87 (95\% CI = 1.67-8.97) for being readmitted within 30 days compared to those who had high adherence. Patients that were not using ACEI or ARB had an OR of 2.40 (95\% CI = 1.09-5.31) for being readmitted within 30 days $(p=0.030)$. Patients having pleural effusion had an OR of 3.25 ( $95 \% \mathrm{CI}=1.44-7.32)$ for being readmitted within 30 days $(p=0.004)$.

Multivariate analysis, seen in Table 3, shows independent factors for early $\mathrm{HF}$ readmission were poor medication
TABLE 2: Clinical characteristics of patients readmitted for heart failure $(N=136)$.

\begin{tabular}{|c|c|c|c|c|c|}
\hline \multirow[b]{2}{*}{ Characteristics } & \multicolumn{3}{|c|}{ Readmission } & \multirow[b]{2}{*}{$\chi^{2}$} & \multirow[b]{2}{*}{$p$ value } \\
\hline & $\begin{array}{l}\text { Total } \\
n(\%)\end{array}$ & $\begin{array}{c}\leq 30 \\
\text { days } \\
n(\%)\end{array}$ & $\begin{array}{c}>30 \\
\text { days } \\
n(\%)\end{array}$ & & \\
\hline NYHA III & $\begin{array}{c}72 \\
(52.9)\end{array}$ & $20(58.8)$ & $52(51.0)$ & 4.02 & 0.866 \\
\hline Elevated SBP & $\begin{array}{c}50 \\
(36.8)\end{array}$ & $15(44.1)$ & $35(34.3)$ & 2.17 & 0.223 \\
\hline Elevated DBP & $\begin{array}{c}42 \\
(30.9)\end{array}$ & $13(38.2)$ & $29(28.4)$ & 1.49 & 0.244 \\
\hline Poor capillary refill & $13(9.6)$ & $7(20.6)$ & $6(5.9)$ & 6.37 & 0.017 \\
\hline Murmur & $\begin{array}{c}47 \\
(34.6)\end{array}$ & $13(38.2)$ & $34(33.3)$ & 0.27 & 0.603 \\
\hline Leukocytosis & $23(16.9)$ & $6(17.6)$ & $17(16.7)$ & 0.45 & 0.951 \\
\hline Anaemia & $26(19.1)$ & $8(23.5)$ & $18(17.6)$ & 0.57 & 0.450 \\
\hline eGFR $<60$ & $\begin{array}{c}78 \\
(57.4)\end{array}$ & $18(52.9)$ & $60(58.8)$ & 0.36 & 0.548 \\
\hline Pleural effusion & $41(30.1)$ & $17(50.0)$ & $24(23.5)$ & 8.48 & 0.004 \\
\hline Pneumonia & $\begin{array}{c}63 \\
(46.3)\end{array}$ & $15(44.1)$ & $48(47.1)$ & 0.08 & 0.766 \\
\hline Atrial fibrillation & $\begin{array}{c}32 \\
(23.5)\end{array}$ & $8(23.5)$ & $24(23.5)$ & 0.05 & 0.815 \\
\hline Reduced EF & $\begin{array}{c}52 \\
(38.2)\end{array}$ & $17(50.0)$ & $35(34.3)$ & 2.65 & 0.103 \\
\hline Preserved EF & $60(44.1)$ & $13(38.2)$ & $47(46.1)$ & 0.63 & 0.425 \\
\hline LV dilatation & $\begin{array}{c}38 \\
(27.9)\end{array}$ & $10(29.4)$ & $28(27.5)$ & 0.06 & 0.799 \\
\hline $\begin{array}{l}\text { Mitral } \\
\text { regurgitation }\end{array}$ & $70(51.5)$ & $20(58.8)$ & $50(49.0)$ & 0.98 & 0.322 \\
\hline
\end{tabular}

adherence $(\mathrm{OR}=3.78,95 \% \mathrm{CI}=1.55-9.20, p=0.003)$ and pleural effusion $(\mathrm{OR}=3.12,95 \% \mathrm{CI}=1.29-7.52, p=0.011)$.

\section{Discussion}

This study found that among all HF admissions, 38.5\% were readmissions. Studies in the United States (US) have shown readmission rates for $\mathrm{HF}$ ranging from $14.2 \%$ to $30 \%$ [18-21], whereas studies in Uganda and Nigeria reported readmission rates of $31.4 \%$ and $35.6 \%$, respectively $[22,23]$. 
TABLE 3: Multivariate analysis of risk factors for heart failure readmission $(N=136)$.

\begin{tabular}{|c|c|c|c|c|}
\hline Characteristics & Unadjusted OR (95\% CI) & $p$ value & Adjusted OR (95\% CI) & $p$ value \\
\hline Unemployed & $2.388(1.029-5.541)$ & 0.043 & $2.171(0.855-5.513)$ & 0.103 \\
\hline Poor medication adherence & $3.877(1.676-8.970)$ & 0.002 & $3.787(1.559-9.200)$ & 0.003 \\
\hline Absence of ACEI or ARB & $2.406(1.090-5.313)$ & 0.030 & $1.960(0.827-4.645)$ & 0.126 \\
\hline Pleural effusion & $3.250(1.441-7.329)$ & 0.004 & $3.126(1.299-7.520)$ & 0.011 \\
\hline
\end{tabular}

The higher readmission rates observed in African settings could be due to difference in health care and number of health care personnel. Low resourced hospitals in low and middle-income countries, as compared to high resourced hospitals in the developed countries, might have higher readmission rates because of financial and clinical reasons such as cardiac capabilities and nurse staffing [24]. Hospitals with low physician volumes had a higher readmission and mortality rate than hospitals with high physician volume [25]. This problem is evident in Tanzania as the doctorpatient ratio is estimated to be $1: 20,000$ [26].

In our study, $52.9 \%$ of HF patients with readmission had NYHA class III. In other settings, the predominant NYHA grade were as follows: in the US, $67 \%$ had NYHA class II [21]; in Nigeria, 52.2\% were class III [23]; in Ethiopia 73.6\% were class IV [27]; and prior research in Tanzania found that $34.0 \%$ were class IV [28]. This shows that HF patients in developing countries present to health centres with later-stage symptoms. These late-stage presentations are likely due to poverty, distance from the hospital, and quality of health care services [29].

Employment status was associated with early readmission $(p=0.043)$. In the US, a study found unemployment to have 2.6 higher odds of being readmitted early ( $p$ 0.013 ) [30]. Unemployment may impair a patient's ability to afford therapies, thereby increasing their risk for decompensation and readmission [31, 32].

This study found that poor medication adherence had 3.8 higher odds for early readmission $(p=0.002)$. A similar study conducted in the US likewise showed that poor adherence to HF medication was associated with 1.7 higher odds of being readmitted early $(p=0.03)$ [30]. This study was only able to detect a difference in readmissions between high adherence and poor adherence patients. It remains unclear whether moderate adherence is better than low adherence. It is plausible there is no significant difference in 30 day readmissions between patients who have poor and moderate adherence, and that the key factor is simply whether or not they are highly adherent [33].

Atrial fibrillation was observed in $23.5 \%$ of $\mathrm{HF}$ readmissions, similar to studies by Dunlay and Fonarow, which observed AF in $26.6 \%$ and $25.2 \%$, respectively [ 20,34$]$. This relatively high rate could be due to the degree of control of $\mathrm{AF}$ and control of left atrial pressures which may influence the frequency of AF. Therefore, a rate control strategy is preferred over rhythm control, especially in HF [35].

Among the patients readmitted for HF, 44\% had HFpEF, similar to findings in the US and Spain, where HFpEF constituted $46 \%$ and $53.7 \%$, respectively, of $\mathrm{HF}$ readmissions $[36,37]$. The incidence of HFpEF is on the rise due to the variety of aetiologies that contribute towards diastolic dysfunction [38]. In theory, diastolic dysfunction is induced by progressive pressure overload, secondary concentric ventricular hypertrophy, microvascular inflammation, interstitial fibrosis, and myocardial remodelling. But studies have shown that there is no adequate therapy to treat HFpEF $[14,39]$.

The use of ACEI or ARB has been shown to be effective in treating HF. This study shows absence of either of these medications increased odds for early readmission $(p=0.030)$. The negative effects caused by angiotensin II are reduced by ACEIs leading to dilation of blood vessels, ventricular remodelling, endothelial protection, and myocardial mass or fibrosis reduction. The mechanism may explain why ACEIs might be superior to ARBs in reducing decompensation $[40,41]$. The clinical benefit of starting the medications early is likely mediated by their favourable hemodynamic effects on ventricular remodelling and disease progression [42]. Although the dose of medication therapy was not measured in this study, patients treated with less than the recommended dose of ACEI or ARB seem to be at a higher risk of readmission compared to those on target doses [43].

Pleural effusions were associated with early readmission as those who presented with pleural effusion had 3.25 higher odds of being readmitted early. Pleural effusions occur when increased pulmonary capillary pressure lead to increased interstitial fluid in the lung. The main treatment is loop diuretics, but the overall cardiac function needs to be improved using effective therapy [44].

The significant independent factors for early readmission were poor medication adherence and having pleural effusion. Poor medication adherence favours disease progression hence would increase the odds of readmission. The presence of pleural effusion may indicate poor HF control. However, this cannot determine whether the effusions were present on the previous admissions. Therefore, the effusions may be new due to worsening HF or may have already been present indicating poor control.

\section{Conclusion}

Heart failure was widely prevalent in our setting. One third of $\mathrm{HF}$ admissions were readmitted and a quarter of these readmissions were within 30 days after discharge. Readmission was strongly associated with unemployment, absence of ACEI or ARBs, poor medication adherence, and pleural effusion. The burden for HF readmission is high and efforts should be made to decrease the decompensation of HF by emphasizing on medication adherence, adequate medication therapy, improved clinic continuity and retention, controlling comorbidities, and lifestyle modification. This will help reduce disease progression thereby decreasing 
the risk of further decompensation, readmission, and mortality due to HF in north-eastern Tanzania.

\section{Data Availability}

The data used to support the findings of this study are available from the corresponding author upon request.

\section{Conflicts of Interest}

The authors declare that they have no conflicts of interest.

\section{Acknowledgments}

The authors are grateful to the patients' participation in this study.

\section{References}

[1] N. B. Ntusi and B. M. Mayosi, "Epidemiology of heart failure in sub-Saharan Africa," Expert Review of Cardiovascular Therapy, vol. 7, no. 2, pp. 169-180, 2009.

[2] A. Damasceno, B. M. Mayosi, M. Sani et al., "The causes, treatment, and outcome of acute heart failure in 1006 Africans from 9 countries," Archives of Internal Medicine, vol. 172, no. 18, pp. 1386-1394, 2012.

[3] G. S. Bloomfield, F. A. Barasa, J. A. Doll, and E. J. Velazquez, "Heart failure in sub-Saharan Africa," Current Cardiology Reviews, vol. 9, no. 2, pp. 157-173, 2013.

[4] D. Kasper, A. Fauci, D. Longo, S. Hauser, J. L. Jameson, and J. Loscalzo, Harrison's Principles of Internal Medicine, McGraw-Hill Education, New York, NY, USA, 19th edition, 2015.

[5] T. Hunter, J. R. Nelson, and J. Birmingham, "Preventing readmissions through comprehensive discharge planning," Professional Case Management, vol. 18, no. 2, pp. 56-63, 2013.

[6] H. D. Lum, S. A. Studenski, H. B. Degenholtz, and S. E. Hardy, "Early hospital readmission is a predictor of one-year mortality in community-dwelling older Medicare beneficiaries," Journal of General Internal Medicine, vol. 27, no. 11, pp. 1467-1474, 2012.

[7] A. Bianco, A. Molè, C. G. A. Nobile, G. Di Giuseppe, C. Pileggi, and I. F. Angelillo, "Hospital readmission prevalence and analysis of those potentially avoidable in southern Italy," PLoS One, vol. 7, no. 11, 2012.

[8] E. F. Philbin, G. W. Dec, P. L. Jenkins, and T. G. DiSalvo, "Socioeconomic status as an independent risk factor for hospital readmission for heart failure," The American Journal of Cardiology, vol. 87, no. 12, pp. 1367-1371, 2001.

[9] J. Hu, M. D. Gonsahn, and D. R. Nerenz, "Socioeconomic status and readmissions: evidence from an urban teaching hospital," Health Affairs, vol. 33, no. 5, pp. 778-785, 2014.

[10] C. Cook, G. Cole, P. Asaria, R. Jabbour, and D. P. Francis, "The annual global economic burden of heart failure," International Journal of Cardiology, vol. 171, no. 3, pp. 368-376, 2014.

[11] O. S. Ogah et al., "Economic burden of heart failure: investigating outpatient and inpatient costs in Abeokuta, Southwest Nigeria," PLoS One, vol. 9, no. 11, Article ID e113032, 2014.

[12] M. O'Connor, C. Murtaugh, S. Shah et al., "Patient characteristics predicting readmission among individuals hospitalized for heart failure," Medical Care Research and Review, vol. 73, no. 1, pp. 3-40, 2016.

[13] R. E. Hoyt and L. S. Bowling, "Reducing readmissions for congestive heart failure," American Family Physician, vol. 63, no. 8, pp. 1593-1598, 2001.

[14] B. Ziaeian and G. C. Fonarow, "Epidemiology and aetiology of heart failure," Nature Reviews Cardiology, vol. 13, no. 6, pp. $368-378,2016$.

[15] D. E. Morisky, L. W. Green, and D. M. Levine, "Concurrent and predictive validity of a self-reported measure of medication adherence," Medical Care, vol. 24, no. 1, pp. 67-74, 1986.

[16] P. H. Lee, D. J. Macfarlane, T. Lam, and S. M. Stewart, "Validity of the international physical activity questionnaire short form (IPAQ-SF): a systematic review," International Journal of Behavioral Nutrition and Physical Activity, vol. 8, no. 1, p. 115, 2011.

[17] L. K. Riley and J. Rupert, "Evaluation of patients with leukocytosis," American Family Physician, vol. 92, no. 92, pp. 1004-1011, 2015.

[18] J. M. Aranda, J. W. Johnson, and J. B. Conti, "Current trends in heart failure readmission rates: analysis of medicare data," Clinical Cardiology, vol. 32, no. 1, pp. 47-52, 2009.

[19] I. Ranasinghe, Y. Wang, K. Dharmarajan, A. F. Hsieh, S. M. Bernheim, and H. M. Krumholz, "Readmissions after hospitalization for heart failure, acute myocardial infarction, or pneumonia among young and middle-aged adults: a retrospective observational cohort study," PLoS Medicine, vol. 11, no. 9, Article ID e1001737, 2014.

[20] G. C. Fonarow, "Factors identified as precipitating hospital admissions for heart failure and clinical outcomes," Archives of Internal Medicine, vol. 168, no. 8, p. 847, 2008.

[21] N. Okumura, P. Jhund, J. Gong et al., "Importance of clinical worsening of heart failure treated in the outpatient setting: evidence from the prospective comparison of ARNI with ACEI to determine impact on global mortality and morbidity in heart failure trial (PARADIGM-HF)," Circulation, vol. 133, no. 23, pp. 2254-2262, 2016.

[22] S. Okello, O. Rogers, A. Byamugisha, J. Rwebembera, and A. J. Buda, "Characteristics of acute heart failure hospitalizations in a general medical ward in Southwestern Uganda," International Journal of Cardiology, vol. 176, no. 3, pp. 1233-1234, 2014.

[23] M. R. Akpa and O. Iheji, "Short-term rehospitalisation or death and determinants after admission for acute heart failure in a cohort of African patients in Port Harcourt, southern Nigeria," Cardiovascular Journal of Africa, vol. 29, no. 1, pp. 46-50, 2018.

[24] K. E. Joynt and A. K. Jha, "Who has higher readmission rates for heart failure, and why? Implications for efforts to improve care using financial incentives," Circulation: Cardiovascular Quality and Outcomes, vol. 4, no. 1, pp. 53-59, 2011.

[25] J. E. Lee, E. C. Park, S. Y. Jang, S. A. Lee, Y. S. Choy, and T. H. Kim, "Effects of physician volume on readmission and mortality in elderly patients with heart failure: nationwide cohort study," Yonsei Medical Journal, vol. 59, no. 2, pp. 243-251, 2018.

[26] N. Sirili, G. Frumence, A. Kiwara et al., "Retention of medical doctors at the district level: a qualitative study of experiences from Tanzania," BMC Health Services Research, vol. 18, no. 1, p. 260, 2018.

[27] T. B. Abebe, E. A. Gebreyohannes, Y. G. Tefera, and T. M. Abegaz, "Patients with HFpEF and HFrEF have different clinical characteristics but similar prognosis: a 
retrospective cohort study," BMC Cardiovascular Disorders, vol. 16, no. 1, pp. 1-8, 2016.

[28] E. Maro and C. Makule, "Causes of hospital readmission with heart failure at Muhimbili National hospital: Tanzanian experience," Tanzania Medical Journal, vol. 24, no. 1, 2009.

[29] D. Musoke, P. Boynton, C. Butler, and M. Musoke, "Health seeking behaviour and challenges in utilising health facilities in Wakiso district, Uganda," African Health Sciences, vol. 14, no. 4, p. 1046, 2015.

[30] L. Calvillo, King, D. Arnold, K. Eubank et al., "Impact of social factors on risk of readmission or mortality in pneumonia and heart failure: systematic review," Journal of General Internal Medicine, vol. 28, no. 2, pp. 269-282, 2013.

[31] D. Amoah and L. Mwanri, "Determinants of hospital readmission of medical conditions in developing countries," Austin Journal of Public Health and Epidemiology, vol. 3, no. 5, 2016.

[32] R. Wilkinson and M. Marmot, Social Determinants of Health: The Solid Facts, World Health Organization, Geneva, Switzerland, 2003.

[33] O. Z. Rosen, R. Fridman, B. T. Rosen, R. Shane, and J. M. Pevnick, "Medication adherence as a predictor of 30-day hospital readmissions," Patient Preference and Adherence, vol. 11, pp. 801-810, 2017.

[34] S. M. Dunlay, M. redfield, S. Weston et al., "Hospitalizations after heart failure diagnosis. A community perspective," Journal of the American College of Cardiology, vol. 54, no. 18, pp. 1695-1702, 2009.

[35] A. Ahmed, W. Ullah, I. Hussain et al., "Atrial fibrillation: a leading cause of heart failure-related hospitalizations; a dual epidemic," American journal of cardiovascular disease, vol. 9, no. 5, pp. 109-115, 2019.

[36] K. S. Shah, H. Xu, R. Matsouaka et al., "Heart failure with preserved, borderline, and reduced ejection fraction: 5-year outcomes," Journal of the American College of Cardiology, vol. 70, no. 20, pp. 2476-2486, 2017.

[37] E. Santas, E. Valero, A. Mollar et al., "Burden of recurrent hospitalizations following an admission for acute heart failure: preserved versus reduced ejection fraction," Revista Española de Cardiología (English Edition), vol. 70, no. 4, pp. 239-246, 2017.

[38] C. Andersson and R. S. Vasan, "Epidemiology of heart failure with preserved ejection fraction," Heart Failure Clinics, vol. 10, no. 3, pp. 377-388, 2014.

[39] A. R. Harper, H. C. Patel, and A. R. Lyon, "Heart failure with preserved ejection fraction," Clinical Medicine, vol. 18, no. 2, pp. s24-s29, 2018.

[40] C. Tai, T. Gan, L. Zou et al., "Effect of angiotensin-converting enzyme inhibitors and angiotensin II receptor blockers on cardiovascular events in patients with heart failure: a metaanalysis of randomized controlled trials," BMC Cardiovascular Disorders, vol. 17, no. 1, pp. 1-12, 2017.

[41] J. S. Borer, "Angiotensin-converting enzyme inhibition: a landmark advance in treatment for cardiovascular diseases," European Heart Journal Supplements, vol. 9, no. suppl_E, pp. 2-9, 2007.

[42] K. Sanam, V. Bhatia, N. Bajaj et al., "Renin-angiotensin system inhibition and lower 30-day all-cause readmission in medicare beneficiaries with heart failure," The American Journal of Medicine, vol. 129, no. 10, pp. 1067-1073, 2016.

[43] W. Ouwerkerk, A. Voors, S. Anker et al., "Determinants and clinical outcome of uptitration of ACE-inhibitors and betablockers in patients with heart failure: a prospective European study," European Heart Journal, vol. 38, no. 24, pp. 18831890, 2017.

[44] J. M. Porcel, "Pleural effusions from congestive heart failure," Seminars in Respiratory and Critical Care Medicine, vol. 31, no. 6, pp. 689-697, 2010. 